Research Article

\title{
To compare the clinicoradiological outcome of various operative techniques and fixation devices in treatment of subtrochanteric fractures
}

\author{
Pandey N. ${ }^{1}$, Chaurasia D.S. ${ }^{2}$ \\ ${ }^{1}$ Dr. Nirnay Pandey, PGMO cum Senior Resident, Department of Orthopaedics, GMC Chhindwara, ${ }^{2}$ Dr. D. S. \\ Chaurasia, Consultant Orthopaedician, Dev Sudha Nursing Home, Tikamgarh.
}

Corresponding Author: Dr. D. S. Chaurasia, Consultant Orthopaedician Dev Sudha Nursing Home Tikamgarh. E-mail: dr.nirnay@gmail.com, dheerendrac81yahoo.com

\begin{abstract}
Background: There are various operative techniques and fixation devices in treatment of subtrochanteric fractures. The objective of treatment is restoration of optimal functions in the shortest possible time by the safest and most dependable method of treatment. Aim: To compare the clinicoradiological outcome of various operative techniques and fixation devices in treatment of subtrochanteric fractures and its variants. To compare the efficacy of various operative techniques in management of subtrochanteric fractures and its variants. To assess efficiency of various fixation devices in different (A-O) types of subtrochanteric fractrues and to compare our results with those available in literature. Methods: This prospective study was conducted in department of orthopedic surgery, in a span of two years. 38 Cases of subtrochanteric fractures and two types of extensions of subtrochanteric fractures admitted in orthopedics wards and treated by various operative methods were included in the study. Results: Malunion was seen in 3 cases and infection in 2 cases. Excellent and good results were seen in most of the cases fixed with intramedullary devices $35 \%$ \& $39 \%$ respectively) and these using closed techniques (38\% \& 43\% respectively). Majority of excellent result (52\%) were seen in cases fixed with Proximal Femoral Nail. Most of the cases fixed using Dynamic Condylar Screw and Dynamic Hip Screw had fair to good results. Conclusion: Successful management of a case of subtrochanteric fracture is an exercise in balancing mechanical and biological consideration in maximize the likelihood of rapid healing and full restoration of function with minimal risk of complications at the least cost to the patient and society
\end{abstract}

Keywords: Operative techniques, Fixation devices, Subtrochanteric fractures, Dynamic Hip Screw Dynamic Condylar Screw

\section{Introduction}

Management of Subtrochancric Fracture has always posed a challenge to orthopaedic surgeon. The objective of treatment is restoration of optimal functions in the shortest possible time by the safest and most dependable method of treatment. The morbidity and mortality together with prolonged hospital stay made conservative treatment unacceptable and internal fixation with appropriate implant is universally advised. Subtrochanteric. fractures are considered as complex fracture. These are particularly difficult to treat firstly because of constant pull of strong abductors of hip and being a highly stressed region of body with forces In the range of 2000 pounds psi operating along the medial cortex about 1 to $2 \mathrm{cms}$ below lesser trochanter [1]

Manuscript Received: $8^{\text {th }}$ July 2019

Reviewed: $18^{\text {th }}$ July 2019

Author Corrected: $26^{\text {th }}$ July 2019

Accepted for Publication: $31^{\text {st }}$ July 2019
Secondly the subtrochanteric area of femur is mainly composed of cortical bone which is often comminuted in these cases. The vascularity of cortical bone and surface available for healing is less. In vivo these bending force loads the medial cortex in compression and lateral cortex in tension. These forces are not in equilibrium. Strainguage studies show that the compressive strains are considerably greater than tensile strains. This dissimilar loading pattern is of great importance in selecting internal fixation device and in understanding the causes and prevention of failure of internal fixation devices [2]. The loading pattern further emphasizes the importance of integrity of medial column as well as the importance of presenting of implant in tension. This is turn increases axial compression which increases the stability of fixation and restores the fractured fragment as a functional unit. 


\section{Research Article}

These are femoral fractures where fracture occurs below the lesser trochanter to $5 \mathrm{~cm}$. distally in femoral shaft, typically at the junction of trabecular \& cortical bone. (where mechanical stress across the junction is highest in the femur and skeleton as well). Also defined as femoral fracture $\mathrm{b} / \mathrm{w}$ lesser trochanter and isthmus of femoral diaphysis [3]. Solitary subtrochanteric femur fractures has very low incidence, i.e. 5-10\% of all extra capsular hip fractures. These are very often extensions of intertrochanteric or diaphyseal fractures. Subtrochanteric fractures with intertrochanteric component occurs in elderly osteoporotic bones suffering a minor fall, while subtrochantric fractures with diaphyseal component occurs in normal bones of young patients suffering a high energy trauma. [4].

There were many studies like Tencer AF, Johnson KD, Hotz TK, Curtis MJ, Jinnah RH, Estrada LS, Anglen $\mathrm{JO}$, Weinstein $\mathrm{JN}$ but still which technique is best remains controvertial, therefore this study was undertaken to compare the clinicoradiological outcome of various operative techniques and fixation devices in treatment of subtrochanteric fractures and its variants [1-6].

\section{Methodology}

Setting, duration and type of study-This prospective study was conducted in department of orthopedics surgery in a span of two years. 38 Cases of subtrochanteric fractures and two types of extensions of subtrochanteric fractures admitted in orthopedics wards and treated by various operative methods were included in the study.

Selections of case/sampling methods, sample size calculation- Fractures involving the subtrochanteric are were included in the study. Also fractures extending to intertrochanteric and or diaphyseal region were included. Compound and Pathological fractures were not included in the study as their clinicoradiological results differ.

Classification system: A-O classification systems of subtrochanteric fractures was followed

Inclusion criteria, exclusion criteria- The present prospective study comprised of 38 cases of subtrochanteric fractures and is variants classified on the basis of AO-ASIF classification system and accordingly fixed with different intramedullary (23) and extramedullary devices (14) using both open and closed techniques of reduction and 1 case managed by Cemented Bipolar Prosthesis .

Surgical procedure- All patients were given preoperative traction and splintage. A definitive postoperative protocol was followed for all the patients in the study, postoperative splintage was given only is special situations and all the patients were followed up for a minimum of 6 months period. All case studied were closed fractures with pathological fractures excluded from the study. Bimodal age distribution of the fracture was confirmed in the present study. Elderly males predominated the study with Male. Female ratio of 1.7.1 with more involvement of right limb. Trivial trauma was major mode of trauma (52\% cases), $13 \%$ cases had associated other bony injuries, while $45 \%$ patients had associated medical illness, most commonly hypertension and diabetes. For the treatment of our cases various intramedullary devices used wereProximal Femoral Nail (14), Interlocking Nail (4), Reconstruction Nail (3) and Kuntscher's Nail (2), while various extramedullary devices used were Dynamic Hip Screw (7), Dynamic Condylar Screw and one case using External Fixator and one case of Cemented Bipolar.

\section{Results}

Majority of fractures (50\%) were type B followed by $42 \%$ cases of type A followed by only $8 \%$ cases of type C. Type B and type A2 fractures were mostly fixed using intramedullary devices whereas majority of type A1 and type $\mathrm{C}$ fractures were fixed by extramedullary devices. Most of the fractures were having diaphyseal extension $(61 \%)$ seen in all age groups and different modes of trauma and mostly fixed with proximal femoral nail (40\%). In majority of cases $(55 \%)$ closed reduction technique was used. The mean opetative time and average blood loss were higher in cases fixed with extramedullary devices (106 min and $200 \mathrm{ml}$ respectively) as compared to cases fixed with intramedullary devices (94 mins and $100 \mathrm{ml}$ respectively) with shortest operative time seen with proximal femoral nail and reconstruction nail causing minimal blood loss. In most of the unstable fractures additional bone grafting was done ( 9 of 38 cases).

Average hospital stay was 18 days in cases fixed with dynamic condylar screw and dynamic hip screw, while it was 14 days for cases fixed with proximal femoral nail. Post-operative splintage was not needed is most of the cases of the present study except for two cases of intramedullary fixtation by kuntscher's nail. 
Research Article

Table-1: Variants of S/Taccording to age of patient.

\begin{tabular}{|c|c|c|c|}
\hline Age & Pure Subtrochanteric & $\begin{array}{c}\text { With Inter- trochanteric } \\
\text { Extension }\end{array}$ & $\begin{array}{c}\text { With Shaft Femur } \\
\text { Extension }\end{array}$ \\
\hline$<$ 20years & 1 & - & 2 \\
\hline 20-40years & 1 & 1 & 4 \\
\hline 40-60years & 2 & 2 & 8 \\
\hline 60-80years & 4 & 3 & - \\
\hline$>80$ years & - & 1 & 4 \\
\hline
\end{tabular}

Table-2: Type of fracture $\&$ fixation device used.

\begin{tabular}{|c|c|c|c|c|c|c|c|c|c|}
\hline & A1 & A2 & A3 & B1 & B2 & B3 & C1 & C2 & C3 \\
\hline DHS & 4 & 2 & & 1 & & & & & \\
\hline DCS & 1 & & & 1 & 2 & & 2 & & \\
\hline ILN & & 1 & & & 2 & & 1 & & \\
\hline Recon & 2 & & & & 1 & & & & \\
\hline 'K'Nail & & & & 1 & 1 & & & & \\
\hline PFN & 1 & 3 & & 8 & 2 & & & & \\
\hline Externalfixator & & 1 & & & & & & & \\
\hline Cementedbipolar & & 1 & & & & & & \\
\hline
\end{tabular}

Table-3: Variant of fracture \& fixation device.

\begin{tabular}{|c|c|c|c|}
\hline & Puresubtrochanteric & $\begin{array}{c}\text { With Inter- } \\
\text { trochantericextension }\end{array}$ & $\begin{array}{c}\text { Withshaftemurextensi } \\
\text { on }\end{array}$ \\
\hline DHS & 2 & 3 & 2 \\
\hline DCS & 1 & 2 & 4 \\
\hline ILN & & & 3 \\
\hline Recon & & 1 & 9 \\
\hline 'K'Nail & 4 & & 2 \\
\hline PFN & 1 & 1 & \\
\hline Externalfixator & & & \\
\hline Cementedbipolar & & & \\
\hline
\end{tabular}

Table-4: Injury operation interval (DAYS)

\begin{tabular}{|c|c|c|c|c|c|c|}
\hline & $0-5$ & $5-10$ & $10-15$ & $15-20$ & $20-25$ & $>25$ \\
\hline DHS & 5 & 1 & --- & --- & 1 & --- \\
\hline DCS & --- & 3 & 2 & --- & 1 & -- \\
\hline ILN & 1 & 1 & 1 & --- & --- & 1 \\
\hline Recon & 1 & --- & 1 & --- & 1 & -- \\
\hline 'K'Nail & --- & --- & 1 & --- & --- & 2 \\
\hline PFN & 7 & 2 & 1 & 1 & --- & -- \\
\hline Externalfixator & 1 & --- & --- & --- & - \\
\hline Cementedbipolar & 1 & --- & --- & - & - \\
\hline
\end{tabular}


Research Article

Table-5: Operative time.

\begin{tabular}{|c|c|c|c|}
\hline & $<\mathbf{1 h r}$ & $\mathbf{1 - 2 h r s}$ & $>\mathbf{2 h r s}$ \\
\hline DHS & --- & 6 & 1 \\
\hline DCS & --- & 6 & --- \\
\hline Externalfixator & 1 & --- & --- \\
\hline ILN & --- & 4 & --- \\
\hline Recon & 1 & 2 & -- \\
\hline 'K'Nail & --- & 13 & -- \\
\hline PFN & 1 & 1 & -- \\
\hline
\end{tabular}

In post-operative period non weight bearing ambulation of patients was started as early as possible (from 3rd day) in cases of intra meduallary fixation because of load sharing nature of the intramedullary devices, whereas it was little delayed in cases fixed by extrameduallary devices. Parital weight bearing was started earlier (within 2 weeks) in almost half of the cases fixed with intramedullary devices while if was delayed (2-4 weeks or more) in majority of cases fixed using extramedullary devices.

All cases were allowed full weight bearing based on clinicoradiological evidence of sound healing of fracture. Full weight bearing was earliest in the cases fixed with proximal femoral nail (3-4 months). Average time of radiological union was significantly less in the cases fixed by intramedullary devices (18 weeks) with fastest union observed in cases fixed with proximal femoral nail (17.1 weeks) as compared to cases treated by extramedullary fixation (20.5 weeks)

Delayed union ( $>6$ months) was found in 3 cases of present study ( 1 case each of dynamic condylar screw, kuntscher's nail and proximal femoral nail). No non-union was seen. Malunion was seen in 3 cases and infection in 2 cases. Excellent and good results were seen in most of the cases fixed with intramedullary devices $35 \%$ \& $39 \%$ respectively) and these using closed techniques ( $38 \% \& 43 \%$ respectively).

Majority of excellent result (52\%) were seen in cases fixed with proximal femoral nail. Most of the cases fixed using dynamic condylar screw and dynamic hip screw had fair to good results

\section{Discussion}

A total number of 18 cases of subtrochanteric tracture were treated by 8 different operative modalities in our series. For selection of operative modality, A-O classification was followed, but in some cases dye to factors like poor bone quality. OT facilities, availability of implants socioeconomic status of patients, diversion from recommendations of A-O ASIF was done. Age or patient varied from 16 to 89 years. Youngest being 16 years and oldest 89 years. Like most of the studies and as per literature available, the present study also showed bimodal age distribution with 19th 39\% patients of 60 80 years followed by $29 \%$ patients of $20-40$ years

In our series trivial trauma was responsible for majority of cases $(52 \%)$ as the present study population was maximum of the age group 60-80 years who are exposed to slip and fall injury more.

High velocity trauma (16\%) and indirect injury (21\%) was mostly seen in young patients in our series. There was no death reported to our series.
Gotze et al compared the loading of ostcosyn thesis of unstable pertochanteric fracture and found that Proximal Femoral Nail could bear the highest load of all devices. In our series majority of cases had diaphyseal extension $(61 \%)$ which is seen in all age groups and various modes of trauma [1].

Mean operative time and operative blood loss were reported higher in extramedullary fixation implants in subtrochnteric fractures. Similar findings was observed in the present series The average hospital stay was 18 days for dynamic hip screw \& dynamic condylar screw cases as compared to 14 days for proximal femoral nail cases. In the present study the average radiological union was significantly faster in cases fixed by intramedullary devices 18 weeks) than the cases fixed by extramedullary devices ( 20.5 weeks).

Tencer AF, Johnson KD et al dida biomechanical comparison of various methods of stabilization of subtrochanteric fractures of the femur. Subtrochanteric 


\section{Research Article}

femoral fractures with and without bony contact were simulated in cadaver specimens, fixed with one of seven different types of intramedullary or plate implants, and tested biomechanically. The implants used were Enders pins, Zickel nail, compression hip screw, AO angled blade plate, and intramedullary locked nail systems of the Klemm-Schellman, Brooker-Wills, and GrosseKempf types.

Femur-implant constructs using intra-medullary devices were a maximum of $5 \%$ as stiff in torsion as intact cadaveric femora tested in the same manner, while plate-fixed fractures were nearly $50 \%$ as stiff. In bending, all devices except the enders pins were $\sim 80 \%$ as stiff as intact femora. Loss of bony contact at the fracture site had little effect on stiffness except in the case of the keyless compression hip screw, where the screw rotated freely in the barrel[2].

Hotz TK et al did their study on minimal invasive treatment of proximal femur fractures with the long gamma nail. They concluded that the LGN is, after appropriate introduction and training, a safe and easy implant for the treatment of complex proximal femur fractures from the trochanteric region to the middle shaft area. The minimal invasive technique with low risks and minimal complications and the possibility of immediate full weight bearing sets a new standard, especially for older patients with osteoporotic bones[3].

Curtis MJ, Jinnah RH et al did a biomechanical study to compare intramedullary and extramedullary fixation of proximal femoral fractures. This study assesses the rigidity and strength of fixation provided by intramedullary and extramedullary devices for proximal femoral fractures. There was no significant difference in the strength of fixation of stable and unstable intertrochanteric fractures between the gamma nail and the hip screw, although the gamma nail provided more rigid fixation [4].

Estrada LS, Volgas DA et al in their study on Fixation failure in femoral neck fractures found that fixation of femoral neck fractures is associated with a higher incidence of complications than any other fracture. The rates of nonunion and avascular necrosis with open reduction and internal fixation continue to be unacceptably high. It may be argued that the most costeffective solution to the femoral neck fracture in the majority of patients is open reduction and internal fixation, with elective conversion, when necessary, to total hip arthroplasty in patients who have a complication [5].
A review was done by Anglen $\mathrm{JO}$ et al in American Board of Orthopaedic Surgery Research Committee. They were trying to see the changing pattern of Nail or plate fixation of intertrochanteric hip fractures. A dramatic change in practice was demonstrated, with the intramedullary nail fixation rate increasing from 3\% in 1999 to $67 \%$ in 2006 .

Regional variation was substantial. Overall, patients managed with plate fixation had slightly less pain and deformity in comparison with those managed with intramedullary nailing, with no significant differences being identified in terms of function or satisfaction. Patients managed with intramedullary nailing had more procedure-related complications, particularly bone fracture [6].

A prospective, randomized study was done by Sadowski C, Lübbeke A et al who did treatment of reverse oblique and transverse intertrochanteric fractures with use of an intramedullary nail or a 95 screw-plate: Implant failure and/or nonunion was noted in seven of the nineteen patients who had been treated with the $95^{\circ}$ screw-plate. Only one of the twenty fractures that had been treated with an intramedullary nail did not heal. The results of study support the use of an intramedullary nail rather than a $95^{\circ}$ screw-plate for the fixation of reverse oblique and transverse intertrochanteric fractures in elderly patients [7].

Comparison of extramedullary and intramedullary devices for treatment of subtrochanteric femoral fractures at tertiary level center was done by Yadav $\mathrm{S}$, Sinha $\mathrm{S}$ et al. The treatment of subtrochanteric fractures is challenging and treatment modalities and implants are constantly evolving. This study attempts to revisit and compare extramedullary vs. intramedullary devices in relatively young population. It was concluded that intramedullary device is a reliable implant for subtrochanteric fractures. It has high rates of union with minimal soft-tissue damage. Intramedullary fixation has biological and biomechanical advantages, but surgery is technically demanding. Gradual learning and patience is needed to make this method truly rewarding [8].

Vidyadhara $\mathrm{S}$ et al did a randomized clinical trial with one and two femoral neck screws with intramedullary nails for unstable trochanteric fractures of femur in the elderly. Although there was no significant difference in the clinical outcome in the two groups, less sliding of the femoral neck screws was noted with two-femoral neck screw configuration. The tip-apex distance was also significantly lower in the two-screw construct 


\section{Research Article}

compared to that of the single screw construct [9]. Functional results using Harris Hip Score in the present study were better in closed techniques of reduction as compared to open one and intramedullary as compared to extramedullary devices. Maximum number of cases done by closed techniques had Good 43\%) and Excellent $38 \%$ ) results with no poor results, while maximum number of cases done by Open techniques had fair results (18\%) with 19\% having. Among individual intramedullary devices $67 \%$ cases of Reconstruction Nail 50\% cases of Interlocking Nail and $36 \%$ cases of Proximal Femoral Nail had Good results, whereas majority of cases of Proximal Femoral Nail had Excellent results (43\%)[10].

Most of the cases using extra medulllary devices were having Fair $(36 \%)$ or Good (29\%) results, with $14 \%$ cases having poor results ( 1 case of dynamic hip screw and dynamic condylar screw each) and $21 \%$ having Excellent results (2 cases of dynamic hip screw and I case of dynamic condylar screw) This was comparable to few series. In Radford series, there were $64 \%$ Excellent or Good results and 27\% Fair results. There was one failure. In series by Kulkarni et. al $77 \%$ of the patients were having Excellent Good results, failure was seen in $23 \%$ of cases [11].

Limitations of the study- Small sample size, observer bias, unicentric study were limitation.

\section{Conclusion}

Successful management of a case of subtrochanteric fracture is an exercise in balancing mechanical and biological consideration in maximize the likelihood of rapid healing and full restoration of function with minimal risk of complications at the least cost to the patient and society. Each subtrochanteric fracture must be individually analyzed and treated in a way that is most likely to succeed in the hands of a responsible surgeon. Compromises are often necessary with certain complications exchanged for increased risk of others.

It can be concluded that AO-ASIF Classification system and recommendations are of great value in deciding the fixation modality and final functional outcomes in these difficult kind of fractures.

\section{What this study adds to the existing knowledge?}

Restoration of posteromedial contact must be the aim of any surgical procedure. Primary bone grafting should be done in very unstable fractures having no posrteromedial bone to bond contact. There is definitely a clear advantage of intramedually devices especially proximal femoral nail over extramedullary devices in terms of better functional results, shorter operative time, less blood loss, faster recovery \& shorter stay at hospital, less chances of infection due to closed approach, better in osteoporotic bones of elderly \& in medically compromised patients, less stress on implant as these are load sharing devices, possibility of dynamization is there if required in future in case of delayed union and good chance of fracture healing by closed techniques of intramedullary fixation as fracture haematoma not disturbed. However, one should keep in mind that intramedullary devices need more competence, are more technically demanding \& require IITV Control.

\section{Author's contribution}

Dr. Nirnay Pandey: Study design, data analysis, and manuscript preparation

Dr D.S. Chaurasia: Study design, data analysis, and manuscript preparation

\section{Conflict of interest: None declared. Funding: Nil, Permission from IRB: Yes Ethical approval: Taken}

\section{References}

1.Götze B, Bonnaire F, Way K, Friedl HP. Resilience of osteosyntheses in unstable per- and subtrochanteric femoral fractures: experimental studies with PFN, gamma nail, DHS / trochanter stabilizing plate, 95condylar plate and UFN / spiral blade. Current traumatol. 1998;28(5):197-204.

2. Tencer AF, Johnson KD, Johnston DW, Gill K. A biomechanical comparison of various methods of stabilization of subtrochanteric fractures of the femur. $J$ Orthop Res. 1984; 2(3): 297-305. doi:10.1002/jor. 1100020312

3. Hotz TK, Zellweger R, Kach KP. Minimal invasive treatment of proximal femur fractures with the long gamma nail: indication, technique, results. J Trauma. 1999; 47(5): 942-5. doi:10.1097/00005373-19991100000023

4. Curtis MJ, Jinnah RH, Wilson V, Cunningham BW. Proximal femoral fractures: a biomechanical study to compare intramedullary and extramedullary fixation. Injury. 1994;25(2):99-104.doi: https://doi.org/10.1016/ 0020-1383(94)90111-2 


\section{Research Article}

5. Estrada LS, Volgas DA, Stannard JP, Alonso JE. Fixation failure in femoral neck fractures. Clin Orthop Relat Res. 2002; (399):110-8. doi:10.1097/00003086200206000-00013

6. Anglen JO, Weinstein JN; American Board of Orthopaedic Surgery Research Committee. Nail or plate fixation of intertrochanteric hip fractures: changing pattern of practice. A review of the American Board of Orthopaedic Surgery Database. J Bone Joint Surg Am. 2008;90(4):700-7. doi: 10.2106/JBJS.G.00517.

7. Sadowski C, Lübbeke A, Saudan M, Riand N, Stern R, Hoffmeyer P. Treatment of reverse oblique and transverse intertrochanteric fractures with use of an intramedullary nail or a 95 degrees screw-plate: a prospective, randomized study. J Bone Joint Surg Am. 2002;84(3):372-81.

8. Yadav S, Sinha S, Luther E, Arora NC, Prasad M, Varma R. Comparison of extramedullary and intramedullary devices for treatment of subtrochanteric femoral fractures at tertiary level center. Chinese $\mathrm{J}$ Traumatol. 2014; 17(3): 1415. doi: https://doi.org/10. 3760/ cma.j. issn.1008-1275.2014.03.004

9. Vidyadhara S, Rao SK. One and two femoral neck screws with intramedullary nails for unstable trochanteric fractures of femur in the elderly-randomised clinical trial. Injury. $2007 \mathrm{Jul}$; 38(7):80614. Epub 2006 . doi:10.1016/j.injury.2006.08.050

10. Radford PJ, Howell CJ. The AO dynamic condylar screw for fractures of the femur. Injury. 1992; 23(2): 89-93.doi: https: //doi. org/10. 1016/0020-1383 (92) 90 039-U

11. Kulkarni SG, Varshneya A, Kulkarni GS, Kulkarni MG, Kulkarni VS, Kulkarni RM. Antegrade interlocking nailing for distal femoral fractures. J Orthop Surg (Hong Kong). 2012; 20(1):48-54. doi:10. 1177/ 230949901202000110

\section{How to cite this article?}

Pandey N, Chaurasia D.S. To compare the clinicoradiological outcome of various operative techniques and fixation devices in treatment of subtrochanteric fractures. Surgical Update: Int J surg Orthopedics. 2019;5(3):176-182. doi:10.17511/ijoso.2019.i03.08. 